\title{
A Review of Personality Type D on Cardiovascular Disease Patients
}

\section{Gratsia Viktoria Fernandez, Rahmatul Fitriyah, Wahyu Sukma Samudera, Hidayat Arifin and Shenda Maulina Wulandari}

Faculty of Nursing, Universitas Airlangga, Surabaya, Indonesia

\begin{abstract}
Introduction: The type of personality that a patient has as a psychosocial factor has been associated with the incidence and progression of cardiovascular disease. The aim of the study was to review the evidence and correlation between personality type and the development of cardiovascular disease.

Methods: Articles were searched for using the PRISMA approach in the CINAHL, Science Direct and Scopus databases, limited to the last 5 years. The articles were from 2013 to 2018 and the language used was English. The studies focused on personality type D and cardiovascular disease, and the participants were above 18 years old.

Results: Most of the findings of the studies showed that individuals with a type D personality have a relationship with more severe heart disease, which relates to several physiological factors, namely emotion increase, anxiety, stress, biological factors, cognitive decline and the decrease in quality of life.

Conclusion: Individuals with type D personality have a higher level of stress, significant blood pressure, higher pulse and they experience an increase in cortisol compared to non-type D personalities. Individuals who suffer from cardiovascular disease with personality type D tend to experience a more severe progression of the condition of cardiovascular disease.
\end{abstract}

\section{ARTICLE HISTORY}

Received: Dec 26, 2019

Accepted: Dec 31, 2019

\section{KEYWORDS}

personality; type D; cardiovascular disease

\section{CONTACT}

Gratsia Viktoria Fernandez

$\triangle$ gratsia.victoria.fernandez2018@fkp.unair.ac.id

$\supseteqq$ Faculty of Nursing, Universitas

Airlangga, Surabaya, Indonesia

Cite this as: Fernandez, G. V., Fitriyah, R., Samudra, S., Arifin, H., \& Wulandari, S. M. (2019). The Current Information on Personality Type D with Cardiovascular Disease: A Systematic Review.Jurnal Ners, 14(3si), 45-49. doi:http://dx.doi.org/10.20473/in.v14i3(si).16976

\section{INTRODUCTION}

Cardiovascular disease is one of the main causes of death globally. The development of cardiovascular disease is caused by accompanying risk factors that are not immediately handled. In some of the research findings, it was found that there was a relationship between personality type and the development of cardiovascular disease (Du et al., 2016).

The development of heart disease has a link with the characteristics of a person's personality. In the case of cardiovascular disease, psychological factors play a crucial role in the development of the disease condition. It is revealed by the results of a previous study that the level of depression and anxiety in a person with cardiovascular disease tends to be higher compared to another person without cardiovascular disease (Du et al., 2016) . Likewise, psychosocial factors such as poor social support and low socioeconomic status also have an influence or relationship with the risk of cardiovascular disease (Bekendam et al., 2018).

The increase of the cardiovascular disease risk mainly has a relationship with the social barriers possessed by a person, including being unable to interact with others. This makes the person tend to have self-restraint or they are not able to express their negative emotions. This can later lead to a disrupted physiological immune system (OgińskaBulik, 2014). Type D stands for distressed and it refers to a set of personality traits that involve things like feelings of worry, sadness, irritability, a pessimistic outlook, negative self-talk, an avoidance of social situations, a lack of self-confidence, fear of rejection, appearing gloomy and hopelessness. 
Based on the results of several previous metaanalysis studies, it has been shown that someone with type D personality had twice the risk of worsening cardiac events and death in the context of patients with coronary heart disease (Kupper \& Denollet, 2016). In this study, the researchers wanted to see the extent of the correlation between personality type D and the development of cardiovascular disease viewed in terms of psychology, biology and physiology.

\section{MATERIALS AND METHODS}

This study used a systematic review design, with a questioning search focused on "What is the correlation between personality type D with the development of cardiovascular disease?" Trials were included in the review identified through the electronic database searches conducted from the earliest available time through to March 2014 in the following databases: Cinahl, Science Direct, Ebsco and Scopus using the keywords "Personality type" and "cardiovascular disease". The inclusion criteria in this review were both experimental studies and nonexperimental studies; studies that were published from March 2013 to 2018; where the participants were above 18 years old; where the language used was English and where they focused on personality type D and cardiovascular disease. The exclusion criteria were studies that did not involve patients with cardiovascular disease and that did not have information on personality type.

Based on the literature research from four databases (Science Direct, Ebsco, Cinahl, and Scopus, there were a total of 1,675 papers. The detailed identification process of the paper selection can be seen in Figure 1. This study reviewed 15 chosen articles. The articles came from Sweden, Turkey, Italy, Swiss, the Netherlands, Germany, Belgium, Spanish, America, China, and Poland.

\section{RESULTS}

The review results show the factors caused by someone having a type D personality as presented in Table 1. Based on Table 1, the researchers were able to determine the factors found in someone with a type D personality. There were two dominant factors found, namely increased stress and increased anxiety. Other factors found were increased emotions, reduced cognitive function, endothelial dysfunction, increased lipids, increased c-reactive point levels, increased superoxide macrophage anions, increased troponin I, increased myocardial infarction, increased stress response, increased stress response, decreased influence positive, decreased quality of life and an increased risk of heart events.

The measuring instrument used in assessing the personality types was DS14, made up of 14 items consisting of 7 negative affective components (NA) and 7 components of social inhibition (SI) coupled with the PHQ-9 (Patient Health Questionnaire) questionnaire, BDI-II (Beck Depression Inventory-

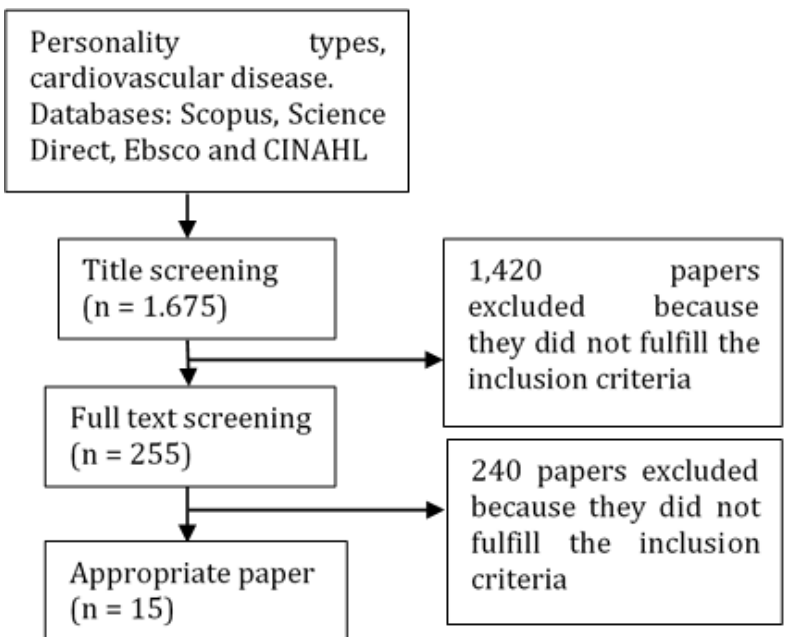

Figure 1. Flowchart of the research on personality type with cardiovascular disease

Second Edition) and CSSS (Chronic Stress Screening Scale) to measure depression. Anxiety was measured using GAD-7 (Generalized Anxiety Disorder Scale), the Spielberger State-Trait Anxiety Inventories (STAII and STAI-II), and the Health Anxiety Inventory (HI). Endothelial function was assessed using the flowmediated dilation (FMD) of the brachial artery, cognitive function was measured using the ToL (Tower of London), positive effects were measured using the GMS (Global Mood Scale), and satisfaction from and with life was evaluated using the Life Satisfaction Questionnaire and Blood Biomarker Measurements.

\section{DISCUSSION}

A person with type D personality has a higher level of stress compared to other personality types. This was found in the study of Du et al, whose results showed that the respondents with type D personality had higher stress and anxiety levels and higher negative coping scores than those for positive coping (Du et al., 2016). Furthermore, in a study conducted by Annagur et al patients with type D personalities had a higher frequency of hypertension, stress and psychiatric disorders, including depression and anxiety than nonType D personality patients (Annagur, Demir, Avci, \& Uygur, 2017). This happens as when stressed, the body will release stress hormones that can narrow the blood vessels and accelerate the heart rate. This causes the blood pressure to easily increase. Increased stress causes a decrease in immune competence (as a consequence of stress hormone secretion) as found in the blood examination of patients with coronary artery disease (CAD) with type D personality.

This results in an increase in the superoxide production of macrophages, resulting in the reactivation of systemic infections and the release of cytokines. The increased superoxide production of macrophages plays an important role in the 
Table 1. List of factors caused by someone having a type D personality

\begin{tabular}{ll}
\hline Authors & Factors caused by someone having a Type D personality \\
\hline Van Montfort et al & Increased emotion \\
Unterrainer et al & Lowered cognitive, Increased stress, Increased anxiety \\
Decreased Flow Mediated Dilation (Endothelial dysfunction) and increased \\
stress \\
Garcia et al(Garcia-retamero et al., & Increased lipid, increased troponin I and increased myoglobin level \\
2016)(Garcia-retamero et al., 2016) & Higher incidence, persistent of smokers, increased stress level, anxiety, and \\
Annagur et al & depression \\
Bekendam et al & Decreased in positive affect \\
Imbalzano et al & Higher incidence of smokers \\
Du et al & Increased stress level, anxiety and re-myocardial infarction \\
Bibbey et al & Increased stress response \\
Steca et al & High levels of anxiety and depression \\
Ogińska-Bulik & Lowered life quality \\
Kupper and Denollet & Increased risk cardiac events \\
Wang et al & Increased c-reactive point level, thinned fibrous cap \\
Leu et al & Increased risk cardiac event (angina and revascularization) \\
Zuccarella-hackl et al & Increased macrophage superoxide anion production and Increased stress \\
\hline
\end{tabular}

pathogenesis of atherosclerosis (Zuccarella-hackl et al., 2016). According to Bibbey et al type D personalities had a higher stress level (Bibbey, Carroll, Ginty, \& Phillips, 2015). People with type D personality have a significant increase in blood pressure, pulse and their amount of cortisol compared to non-type D personalities. Stress is one of the risk factors for cardiovascular disease and it also has an association with increased systolic blood pressure. Stress can trigger the release of catecholamine quickly which will later cause an increase in cardiac output and blood pressure. In addition, stressful conditions will activate the hypothalamus-pituitary-adrenal which will release adrenal glucocorticoids and cortisol. Corticotropinreleasing hormone (CRH) is released by the hypothalamus in response to stress, which then affects the anterior pituitary gland to prompt it to release adrenocorticotrophic hormone (ACTH). This in turn causes the adrenal cortex to release cortisol. The cortisol hormone caused the increased breakdown of protein and fat that will be converted into blood glucose, so this increases the blood sugar level. This increases the risk of cardiovascular disease due to an increase of fatty acid (Bibbey et al., 2015). In the cardiovascular system, cortisol is required to protect the balance of blood pressure, heart function, and blood vessel responses. However, if the amount of cortisol increases, then the protection function of the heart is also impaired.

In type D personality, the level of their emotions will increase, according to Van Monfort et al study (Van Montfort, Mommersteeg, Spek, \& Kupper, 2018). This proved that a person's emotional level is related to a combination where people have type D personalities and bad life experiences. This emotional level can be caused by a negative effect and high social barriers, which will affect their lifestyle. This is also in line with the research conducted by Steca et al, which explained that a person with type D personality, especially men who do not have many friends, has a high level of anxiety and depression, low levels of optimism and self-esteem, and they have a bad lifestyle such as a poor diet, higher fat intake, smoking and poor adherence to treatment (Steca et al., 2016).

According to the research of Wang et al, they showed that there was a relationship between type D personality and susceptibility to coronary plaque (Wang et al., 2016). In type D personality, the respondents tend to have more lipid-rich plaques and macrophages. This is in line with the study of Garcia et al, which revealed that respondents with type D personality who smoked had a high blood pressure systole and thus had poor lipid profile examination results, especially concerning HDL (Garcia-retamero, Petrova, \& Ram, 2016). An increase in lipids is caused by the lifestyle of patients with type D personality who are less healthy, such as a lack of exercise, smoking and not following the recommended diet (Imbalzano et al., 2018). In addition, type D patients often lack social support, which can indirectly contribute to the failure to adopt a healthy lifestyle and lipid control.

Type D personality also affects the quality of life of CVD patients. This was found in a study conducted by Oginska, which proved that the level of quality of life in respondents with type $\mathrm{D}$ personality was significantly lower than all of the components when compared to the respondents with a Non-type D personality (Ogińska-Bulik, 2014). In measuring Type D personality, the social obstacle component had a close correlation with the life quality level. It related to the interactions with other people. The more that people were socially isolated and often hid their emotions, the lower their life quality level was. Accordingly, it was required that psychology rehabilitation should not only have the goal to overcome the stress but it should also increase their openness with other people, thus allowing them to identify and use social support.

Cognitive function also had an influence regarding Type D personality. In the research conducted by Unterainner et al, it was explained that Type D personality had a relation with lower cognitive 
function in the respondents with CVD (Unterrainer et al., 2016). The downgrade of cognitive function was connected with the existence of cardiovascular disease which could lead to a lack of blood supply to the brain. Moreover, people who had a high negative effect from their Type D personality had high stress and a high anxiety level, which would influence their own cognitive function.

The existence of endothelial dysfunction is also related to Type D personality in patients with CVD. This could be proven by the research conducted by Denollet et al, which explained that endothelial dysfunction is the key factor which was able to connect Type D personality with the higher heart attack risk (Denollet et al., 2018). Negative affect and social obstacles could be the factors of endothelial dysfunction in Type D personality. The increase of superoxide anion formation, stress oxide, TNF- $\alpha$, and cortisol were part of a biological pathway where Type D personality contributes to endothelial dysfunction. The stress level of Type D personality also influences endothelial dysfunction.

Type D personality could be a predictive factor for the occurrence of Major Adverse Cardiac Events (MACE) consisting of several components: death, myocardial infarction, Coronary Artery bypass Surgery (CABG), Percutaneous Intervention (PCI) or the development of Coronary Artery Disease (CAD). Based on the research conducted by Kupper and Denollet, it was revealed that there was a correlation between type D personality and MACE. This was indicated by the increased risk when under 70 years old (Kupper \& Denollet, 2016). The biological mechanisms found in the type D personality were related to specific diseases such as the increase of the amount of plaque in the coronary arteries, the increase of macrophage activity, the increase of oxidative stress, endothelial dysfunction and the increase of cortisol, which could be a marker of heart disease risk. In older patients, the risk of death was higher than the risk of the younger patients due to the aging process, increased comorbidity and the decreased medication options. This is also in line with the research conducted by Leu et al, which revealed that type $\mathrm{D}$ personality can be independently associated with an increased risk of future heart events (Leu et al., 2018). In addition, the risk profile of type D personality is even more significant in women, older patients, patients with hypertension and patients with type 2 diabetes. Meanwhile, in a study conducted by Bekendam et al, it was explained that people with a positive affect can avoid cardiovascular disease (Bekendam et al., 2018). Positive affect generally refers to positive mood conditions such as enthusiasm, excitement, happiness, joy and satisfaction. However, further studies are required to prove that positive affect could provide a protective effect against CVD.

\section{CONCLUSION}

Individuals with cardiovascular disease who have a type D personality have a tendency to develop a more severe cardiovascular condition. This is related to several factors that are physiological, psychological and biological. Through the systematic review, the author has tried to identify the disorders caused by type D personality. The physiological and biological disorders include increased lipids, increased troponin I, increased myoglobin, increased c-reactive point level, increased risk of a cardiac event (angina and revascularization, superoxide macrophages increased anion production and decreased endothelial function. The psychological disorders include increased emotions, increased levels of stress, anxiety, decreased positive affect and decreased quality of life. After learning of these factors, it is expected to be a reference to be able to modify the coping mechanisms of people with type D personality so then the disorder can be avoided and minimized. After recognizing the factors, it is expected that the results will a reference to be able to modify the coping mechanisms of people with type D personality so then the interference can be avoided and minimized.

\section{REFERENCES}

Annagur, B., Demir, K., Avci, A., \& Uygur, Ö. (2017). Impact of a Type D Personality on Clinical and Psychometric Properties in a Sample of Turkish Patients With a First Myocardial Infarction. Journal of Psychiatric Practice, 23(1), 3-10. https://doi.org/10.1097/PRA.00000000000002 01

Bekendam, M. T., Kop, W. J., Barzilay, S., Widdershoven, J. W., Aarnoudse, W., Denollet, J., \& Mommersteeg, P. M. C. (2018). The predictive value of positive affect and Type $D$ personality for adverse cardiovascular clinical outcomes in patients with non-obstructive coronary artery disease. Journal of Psychosomatic Research, 104(October 2017), 108-114. https://doi.org/10.1016/j.jpsychores.2017.11.00 3

Bibbey, A., Carroll, D., Ginty, A. T., \& Phillips, A. C. (2015). Cardiovascular and cortisol reactions to acute psychological stress under conditions of high versus low social evaluative threat: Associations with the type D personality construct. Psychosomatic Medicine, 77(5), 599608.

https://doi.org/10.1097/PSY.000000000000019 4

Denollet, J., van Felius, R. A., Lodder, P., Mommersteeg, P. M., Goovaerts, I., Possemiers, N., ... Van Craenenbroeck, E. M. (2018). Predictive value of Type D personality for impaired endothelial function in patients with coronary artery disease. International Journal of Cardiology, 259(2017), 205-210. https://doi.org/10.1016/j.ijcard.2018.02.064

Du, J., Zhang, D., Yin, Y., Zhang, X., Li, J., Liu, D., ... Chen, W. (2016). The Personality and Psychological 
Stress Predict Major Adverse Cardiovascular Events in Patients With Coronary Heart Disease After Percutaneous Coronary Intervention for Five Years. Medicine, 95(15), e3364. https://doi.org/10.1097/MD.000000000000336 4

Garcia-retamero, R., Petrova, D., \& Ram, A. (2016). Type $\mathrm{D}$ personality is related to severity of acute coronary syndrome in patients with recurrent cardiovascular disease. British Journal of Health Psychology, 14, 111-119. https://doi.org/10.1111/bjhp.12196

Imbalzano, E., Vatrano, M., Quartuccio, S., Ceravolo, R., Ciconte, V. A., Rotella, P., ... Mandraffino, G. (2018). Effect of type D personality on smoking status and their combined impact on outcome after acute myocardial infarction. Clinical Cardiology, 41(3), 321-325. https://doi.org/10.1002/clc.22865

Kupper, N., \& Denollet, J. (2016). Explaining heterogeneity in the predictive value of Type D personality for cardiac events and mortality. International Journal of Cardiology, 224, 119-124. https://doi.org/10.1016/j.ijcard.2016.09.006

Leu, H., Yin, W., Tseng, W., Chang, K.-C., Wang, J., \& Wu, C. (2018). Impact of type D personality on clinical outcomes in Asian patients with stable coronary artery disease. Journal of the Formosan Medical Association, https://doi.org/10.1016/j.jfma.2018.08.021

Ogińska-Bulik, N. (2014). Type D personality and quality of life in subjects after myocardial infarction. Kardiologia Polska, 72(7), 624-630. https://doi.org/10.5603/KP.a2014.0066

Steca, P., D'Addario, M., Magrin, M. E., Miglioretti, M., Monzani, D., Pancani, L., ... Greco, A. (2016). A type $\mathrm{A}$ and type $\mathrm{D}$ combined personality typology in essential hypertension and acute coronary syndrome patients: Associations with demographic, psychological, clinical, and lifestyle indicators. PLoS ONE, 11(9), 1-28. https://doi.org/10.1371/journal.pone.0161840

Unterrainer, J., Michal, M., Rahm, B., Hadzibegovic, J., Wild, P. S., Schulz, A., ... Beutel, M. E. (2016). Association of Type D personality with cognitive functioning in individuals with and without cardiovascular disease - The Gutenberg Health Study. International Journal of Cardiology, 214, 256-261.

https://doi.org/10.1016/j.ijcard.2016.03.221

Van Montfort, E., Mommersteeg, P., Spek, V., \& Kupper, N. (2018). Latent profiles of early trauma \& Type D personality: sex differences in cardiovascular risk markers. Comprehensive Psychiatry, 83, 38-45. https://doi.org/10.1016/j.comppsych.2018.02.0 09

Wang, Y., Zhao, Z., Gao, X., Li, L., Liu, G., Chen, W., ... Lin, P. (2016). Type D personality and coronary plaque vulnerability in patients with coronary artery disease: An optical coherence tomography study. Psychosomatic Medicine, 78(5), 583-592. https://doi.org/10.1097/PSY.000000000000030 7

Zuccarella-hackl, C., Känel, R. Von, Thomas, L., Kuebler, P., Schmid, J., Mattle, H. P., ... Wirtz, P. H. (2016). Psychoneuroendocrinology Higher macrophage superoxide anion production in coronary artery disease ( CAD ) patients with Type D personality. Psychoneuroendocrinology, 68, 186193.

https://doi.org/10.1016/j.psyneuen.2016.02.031 